\title{
Transatlantica
}

Revue d'études américaines. American Studies Journal

\section{Henri Cartier-Bresson / Walker Evans : Photographier l'Amérique (1929-1947)}

Fondation Henri Cartier-Bresson, 10 septembre - 21 décembre 2008. Voir la présentation sur le site de la Fondation. Ouvrage associé sous le même titre, avec des textes d'Agnès Sire et Jean-François Chevrier, Göttingen : Steidl, 2008.

\section{François Brunet}

\section{OpenEdition}

Journals

\section{Édition électronique}

URL : https://journals.openedition.org/transatlantica/4304

DOI : $10.4000 /$ transatlantica.4304

ISSN : 1765-2766

\section{Éditeur}

Association française d'Etudes Américaines (AFEA)

\section{Référence électronique}

François Brunet, « Henri Cartier-Bresson / Walker Evans : Photographier l'Amérique (1929-1947) », Transatlantica [En ligne], 1 | 2009, mis en ligne le 05 août 2009, consulté le 23 septembre 2021. URL : http://journals.openedition.org/transatlantica/4304; DOI : https://doi.org/10.4000/transatlantica. 4304

Ce document a été généré automatiquement le 23 septembre 2021.

\section{c)}

Transatlantica - Revue d'études américaines est mise à disposition selon les termes de la licence Creative Commons Attribution - Pas d'Utilisation Commerciale - Pas de Modification 4.0 International. 


\section{Henri Cartier-Bresson / Walker Evans : Photographier l'Amérique (1929-1947)}

Fondation Henri Cartier-Bresson, 10 septembre - 21 décembre 2008. Voir la présentation sur le site de la Fondation. Ouvrage associé sous le même titre, avec des textes d'Agnès Sire et Jean-François Chevrier, Göttingen : Steidl, 2008.

\section{François Brunet}

1 Dans un mot adressé en 2001 à Peter Galassi, conservateur de la photographie au MOMA, Henri Cartier-Bresson, alors âgé de quatre-vingt-treize ans, écrivait plaisamment - mais non pas complaisamment : "If it had not been for the challenge of the work of Walker Evans, I don't think I would have remained a fotographer " («sans le défi constitué par le travail de Walker Evans, je ne crois pas que je serais resté fotographe »). Pour fêter le centenaire de Cartier-Bresson, c'est ce défi - plutôt qu'une ènième rétrospective du grand maître de l'instantané - que la Fondation qui porte son nom a choisi de mettre en scène, en prenant pour objet les regards que portent les deux photographes sur l'Amérique de la Dépression (Evans, avant, pendant et après son bref engagement dans la Farm Security Administration) et de l'après-guerre (CartierBresson lors de son périple américain en 1947).

2 On trouvait ainsi dans cette superbe exposition un grand nombre d'images remarquables de cette Amérique en noir et blanc, généralement fruste, affligée et surtout muette chez Evans, plus animée ou parlante, gaie ou franchement grinçante chez Cartier-Bresson (comparer «Westchester, New York, 1931 » du premier à «Arizona, 1947 » du second). Plus que le sujet Amérique, cependant, resté assez marginal dans l'œuvre du Français alors qu'Evans n'en est pour ainsi dire jamais sorti, ce qui lie ces deux grands maîtres est peut-être d'abord un penchant littéraire, à partir duquel l'un et l'autre ont, chacun à sa façon, "réinventé la photographie » comme l'écrit Jean-François Chevrier. Evans, l'aîné (de cinq ans), est flaubertien et réaliste, amateur d'effacement; Cartier-Bresson est plus romantique, peut-être plus poète, et a 
appris le « hasard objectif » chez les surréalistes. Ensemble, toutefois, ils ont consacré le livre, et plus spécialement le livre co-écrit avec un écrivain, comme le médium par excellence de la photographie d'auteur; par là, au moins, leur couple est fondateur pour la notion même d'auteur en photographie, comme le montraient bien les diverses publications des deux hommes réunies en vitrine. Ce goût partagé pour ce qu'on appellera le photo-essay vient pour une part d'une histoire commune, qui les a très tôt placés du côté du «document» - ils étaient réunis dès 1935 par la galerie de Julien Levy à New York, avec Manuel Alvarez Bravo, pour une exposition de photographie « antigraphique » et « documentaire » c'est-à-dire anti-pictorialiste. Les livres d'Evans (American Photographs, 1938; Let Us Now Praise Famous Men, avec James Agee, 1941) devaient devenir les modèles absolus de l'esthétique du document. Cartier-Bresson, après un essai infructueux de tirer un livre de son voyage américain, reprit clairement et explicitement le modèle du livre photographique, sinon le "style documentaire ", pour The Decisive Moment (1951, titre français Images à la sauvette). De cette histoire commune, enfin, est née, dès les années trente, une estime réciproque qui ne s'est jamais démentie par la suite, et que l'exposition éclairait grâce à un certain nombre de documents, comme l'éloge de «l'œil » de Cartier que publia Walker Evans dans le New York Times en 1950 ( Cartier-Bresson a True Man of the Eye »).

3 Mais le véritable propos de l'exposition était, comme on s'y attend, de susciter une compréhension du « défi » Evans aux yeux de Cartier-Bresson, dont le point de vue à la fois photographique et culturel est ici logiquement privilégié, comme en témoigne d'ailleurs la réception critique (voir par exemple le commentaire de Louis Mesplé sur rue89). La scénographie, sobre mais réfléchie, évitait parfaitement la formule jeu de société qui eût consisté à aligner de sèches paires Cartier / Evans et proposait d'amples séries de l'un et de l'autre, pour Evans des images souvent connues des années trente, pour Cartier, en revanche, beaucoup d'images peu connues, voire inédites, tirées de son voyage américain.

4 Le spectateur passait ainsi d'ensemble à ensemble, déterminés par des critères tantôt géographiques et chronologiques tantôt visuels, et, séjournant chez l'un ou chez l'autre, s'habituait à son regard ou à sa technique avant de se transporter dans l'univers du second. Là où, au MOMA en 2001, Walker Evans and Company avait insisté au contraire sur l'influence et l'héritage du photographe américain, la mise en scène volontairement plate de la Fondation HCB offrait l'occasion, somme toute assez rare, d'explorer en profondeur, par la contemplation un tant soit peu prolongée de deux œuvres ou segments d'œuvres, la notion si souvent galvaudée de regard photographique.

On prend donc conscience, dans la différence de deux regards, de l'existence du regard. Comme l'écrit Michel Poivert dans un commentaire très fin, Cartier-Bresson « vise » en instantanéiste, et « ce qui est donné à voir porte en soi la magie du furtif soustrait au flot continu du monde »; Walker Evans, plus « archaïque » que classique, « compose sur le dépoli plus qu'il ne vise, il ajuste, il revient, il condense le temps ». Tous deux construisent, certes, mais il s'agit plutôt d'espace chez Evans et de figure chez Cartier (voir par exemple «Memphis, Tennessee, $1947 »$ ). On pourrait multiplier ce genre de remarques, en notant par exemple que là où les vues frontales d'Evans interdisent en général une lecture narrative (tout en reflétant l'histoire dans une veine parfois proche de la satire), les photos de Cartier sont très souvent construites sur une mobilité, une tension, une bipolarité (ne serait-ce qu'entre premier plan et arrière-plan), qui autorisent cette lecture et peuvent même suggérer l'anecdote. On se reportera, pour 
une élucidation pénétrante et approfondie de cette confrontation, au remarquable essai de Jean-François Chevrier contenu dans l'élégant ouvrage au format carré qui accompagne l'exposition. De fait, toute une panoplie d'oppositions peut être convoquée à propos du vis-à-vis Cartier / Evans - Leica / chambre, composition / enregistrement, instant / durée, perspective / frontalité, temps / espace, voire monde / Amérique - qui tend, pour Chevrier, à inscrire le photographe français dans un réalisme plus « universaliste » que celui d'Evans, en un sens borné par son horizon américain. Il y a là, sans doute, une «limite» au «dialogue » entre les deux hommes ${ }^{1}$, sans que pour autant soit jamais défaite, dans la visite de l'exposition, l'intuition d'une parenté profonde qui trouve son origine dans un refus très moderne (et très masculin) de la fioriture et l'affirmation concomitante du photographe comme auteur.

\section{NOTES}

1. Jean-François Chevrier, « Un dialogue ? », in Henri Cartier-Bresson / Walker Evans, Photographier l'Amérique 1929-1947, Steidl, 2008, p. 47.

\section{INDEX}

Thèmes : Trans'Arts 